\title{
Meta
}

Journal des traducteurs

Translators' Journal

\section{Nouveaux dictionnaires, nouveaux rapports avec les utilisateurs}

\section{John Humbley}

Volume 47, numéro 1, mars 2002

URI : https://id.erudit.org/iderudit/007994ar

DOI : https://doi.org/10.7202/007994ar

Aller au sommaire du numéro

Éditeur(s)

Les Presses de l'Université de Montréal

ISSN

0026-0452 (imprimé)

1492-1421 (numérique)

Découvrir la revue

Citer cet article

Humbley, J. (2002). Nouveaux dictionnaires, nouveaux rapports avec les utilisateurs. Meta, 47(1), 95-104. https://doi.org/10.7202/007994ar
Résumé de l'article

Les dictionnaires, surtout spécialisés, sont de mieux en mieux adaptés aux besoins des utilisateurs, grâce aux progrès réalisés en informatique, qui ouvrent de nouveaux horizons. Nous examinons ici quelques-unes de ces nouvelles possibilités, ainsi que les réactions des lexicographes et des terminologues. Certains de ces nouveaux besoins sont illustrés ici dans les domaines de l'enseignement, du droit et de l'administration, et dans les entreprises ; nous analysons enfin quelques solutions proposées sous forme de nouveaux types de dictionnaire. 


\title{
Nouveaux dictionnaires, nouveaux rapports avec les utilisateurs
}

\author{
JOHN HUMBLEY \\ Université Paris 13, Paris, France
}

\begin{abstract}
RÉSUMÉ
Les dictionnaires, surtout spécialisés, sont de mieux en mieux adaptés aux besoins des utilisateurs, grâce aux progrès réalisés en informatique, qui ouvrent de nouveaux horizons. Nous examinons ici quelques-unes de ces nouvelles possibilités, ainsi que les réactions des lexicographes et des terminologues. Certains de ces nouveaux besoins sont illustrés ici dans les domaines de l'enseignement, du droit et de l'administration, et dans les entreprises; nous analysons enfin quelques solutions proposées sous forme de nouveaux types de dictionnaire.
\end{abstract}

\begin{abstract}
Dictionaries, and more particulary specialist dictionaries, are ever increasingly being adapted to correspond more closely to users' needs. This has been made possible by advances in information technology which open up new possibilities, some of which are reviewed here, along with the responses from lexicographers and terminologists. Illustrations are given of differing user needs, in education, law/administration and industry, as well as several solutions provided in the form of new kinds of dictionaries.
\end{abstract}

\section{MOTS-CLÉS/KEYWORDS}

dictionnaire, terminologie, lexicographie, besoins des utilisateurs

Le rapport entre le dictionnaire et ses utilisateurs est en train d'évoluer sous la pression conjuguée d'une demande plus exigeante et d'une offre stimulée par les nouveaux outils, avant tout informatiques. Cependant, plus qu'à une évolution purement technique, on assiste à un changement de perspective. Le lexicographe d'autrefois, comme d'ailleurs le terminologue, visait un produit "en soi», un dictionnaire ou une terminologie qui rende compte d'un état de langue ou d'un domaine de spécialité considéré comme préexistant. Aujourd'hui, le terminologue comme le lexicographe dialoguent de plus en plus avec l'utilisateur afin de mieux répondre à ses besoins. Car, du côté de l'utilisateur, on constate de nouvelles exigences, que les possibilités informatiques rendent désormais possibles, à condition que les lexicographes et les terminologues se mettent à son écoute.

Nous nous proposons d'examiner d'abord quelques aspects de la relation qui lie le lexicographe ou le terminologue à son destinataire, tout en nous interrogeant sur des différences éventuelles entre les démarches de ces deux disciplines en matière de prise en compte de l'utilisateur, et puis de donner quelques exemples de produits dictionnairiques ou terminologiques faits sur mesure. Ce faisant, nous adoptons une définition très large de ce qu'est un dictionnaire, mais il nous semble que cet abus de langage apparent est le prix à payer pour l'innovation, car non seulement le dictionnaire de demain ne ressemblera pas à celui d'hier, mais en plus il sera multiforme. 
Si nous considérons l'évolution des dictionnaires depuis le Moyen-Âge, nous constatons un éloignement grandissant entre le concepteur et l'utilisateur. On prétend généralement que l'ancêtre du dictionnaire moderne est le glossaire des moines-copistes, qui inscrivaient en marge de leurs manuscrits les équivalents en langue vulgaire des mots difficiles du texte latin, précurseur du glossaire de nos jours. A cette époque-là, les créateurs de dictionnaires étaient aussi des utilisateurs ou du moins ils étaient très proches de ceux-ci. Par la suite, surtout à partir de l'invention de l'imprimerie, on assiste à une division du travail qui éloigne l'utilisateur du concepteur de dictionnaire, surtout dans le cas des grands dictionnaires nationaux, dont l'apothéose est sans doute le dictionnaire de Grimm. En effet, l'utilisateur aura dû attendre plus de cent ans pour passer de la lettre A à la lettre Z. Plus récemment, des efforts importants ont été faits pour rapprocher le lexicographe des utilisateurs, mais de façon inégale selon les types de dictionnaire.

Compte tenu de la complexité de la société moderne, il n'est pas facile de cerner les attentes des utilisateurs de dictionnaires. P. Bogaards a présenté à l'AILF de 1993 un recensement de différentes enquêtes menées sur l'utilisation que font les usagers de différents types de dictionnaires. «People, mostly secondary school or university students, do not seem to be able to profit fully from the wealth modern dictionaries offer them.» (Bogaards 1993: 697). Les dictionnaires d'apprentissage - surtout de la langue maternelle - étaient majoritaires dans son étude, qui ne tenait pas compte des dictionnaires de spécialité, qui nous intéressent ici.

En fait, cette lacune avait déjà été comblée grâce à une étude approfondie menée au Danemark dans le cadre d'un projet d'envergure sur la traduction de textes spécialisés - appelé en Scandinavie la conférence de Sandbjerg (Jakobsen, 1992). Le but de cette enquête, par ailleurs très riche en renseignements, n'était pas de proposer des améliorations aux dictionnaires existants à la lumière des difficultés rencontrées lors de leur utilisation, mais de comprendre comment les traducteurs (professionnels ou apprentis) se comportaient devant l'outil lexicographique. On peut néanmoins retenir un certain nombre de résultats, qui fournissent des pistes de recherche sur l'utilisation effective des dictionnaires, décelées grâce à des techniques telles que le questionnaire direct, et surtout le « carnet de bord» du traducteur, très révélateur de la démarche de l'utilisateur face à un problème de langue. On constate que, souvent, les traducteurs n'emploient pas le bon dictionnaire tout simplement parce qu'ils ne le connaissent pas. Le problème de l'utilisation commence donc bien avant que l'utilisateur ait le dictionnaire sous les yeux et replace le dictionnaire dans le cadre plus général de la documentation. L'enquête révèle également que les traducteurs préfèrent ce qu'ils connaissent: les dictionnaires généraux aux dictionnaires spécialisés, les dictionnaires anglais aux dictionnaires français...

Mais on n'a pas nécessairement besoin de réaliser des enquêtes pour connaître les exigences des utilisateurs. Certains s'expriment spontanément. Dans le domaine francophone, on a assisté, dès le début des années 1990, à tout un mouvement vers la prise en compte de la "phraséologie» dans les dictionnaires de spécialité. Cette prise de conscience était provoquée par l'intervention de très nombreux traducteurs qui reprochaient aux dictionnaires de spécialité et aux banques de terminologie de limiter les informations aux termes et à leur description conceptuelle et de passer sous silence tous les problèmes liés à «la mise en discours». On peut se demander si la phraséologie était suffisante comme réponse à ce défi, mais ceci est un autre débat. 
La contribution des Scandinaves au débat sur la définition des besoins de l'usager ne se limite pas aux enquêtes. Elle comprend aussi une polémique à peu près absente dans les pays latins, et qui oppose terminologues et lexicographes. Lorsque le premier manuel de lexicographie spécialisée est paru en 1993, (Bergenholtz et Tarp 1993), plus d'un lecteur a remarqué que la terminologie était à peu près absente et donc de la démarche. Cette absence traduit une coupure radicale entre les tenants de la tradition lexicographique, adaptée aux dictionnaires de spécialité, et ceux de la terminologie, fortement influencés par les travaux de normalisation. L'intérêt du débat pour notre propos est de mieux situer l'utilisateur en lexicographie et en terminologie.

Le chef de file des lexicographes, Henning Bergenholtz, co-auteur du manuel de terminologie spécialisée, aujourd'hui traduit en anglais, ainsi que d'un dictionnaire de génie génétique (Kaufmann et Bergenholtz 1992), porte des accusations sérieuses à l'adresse des terminologues.

Il commence par renvoyer dos à dos la terminologie et la lexicographie:

"In der Lexicographie, darunter der Fachlexikographie, war der Benutzer vor kurzem ein oft erwähntes, aber im Grunde unbekanntes Wesen. Erst in den letzten 15 Jahren hat hier eine entscheidended Neuorientierung stattgefunden. Auch in der terminologischen Tradition kann ein fehlended Interesse für eine Benützerforschung festgestellt werden.» Bergenholtz 1995: 55. (En lexicographie, dont la lexicographie spécialisée, l'utilisateur était jusqu'à très récemment un être souvent évoqué, mais en réalité inconnu. On constate une nouvelle orientation allant nettement dans ce sens depuis seulement une quinzaine d'années. Le même manque d'intérêt pour les études sur l'utilisation peut être constaté également dans la tradition terminologique.)

La nouvelle orientation dont parle Bergenholtz comporte donc la prise en compte des besoins des utilisateurs, traduits pour le lexicographe en termes de fonctions lexicographiques. Mais, toujours selon Bergenholtz, les terminologues tardent à faire leur mue, et ceci, poursuit-il, pour des raisons idéologiques: "Dies wird teilweise zum Prinzip erklärt, indem hindurch die wichtigste Unterscheidung zur Fachlexikographie gesehen wird. Während die Fachlexikographie nur eine begrentzte Benutzung - eben gerade die Berücksichtigung der vorgesehenen Benutzersitutationene - zulasse, habe die Terminographie nicht dies Beschränkung. Umgekehrt könne man aus einer Wissensbank nach Bedarf bestimmte "Wissensprofile » herausziehen (Picht 1992: 30). » ... Bergenholtz 1995: 55. [Ceci s'explique en partie par une des différences fondamentales entre la terminologie et la lexicographie de spécialité. Tandis que celle-ci n'aurait qu'un emploi limité par les situations d'utilisation prévues, la terminographie n'aurait pas cette limitation. Au contraire, on peut toujours extraire des informations selon le profil de la requête dans une banque de connaissances (Picht 1992: 30)]

La banque de données, typique de la présentation terminologique, est ici présentée comme banque de connaissances terminologiques, dans laquelle chacun peut puiser ce qu'il veut. Bergenholtz suppose que le contenu de ces bases représente la «vérité vraie» sur l'objet de la connaissance en question et que leur but est de découvrir cette réalité et de la présenter. Nous allons voir que cette approche, qui est parfois celle de certains terminologues, est bien trop simpliste, et qu'elle doit faire l'objet d'aménagements considérables. Bergenholtz, du moins dans l'article de 1995, ne conteste pas la possibilité de réaliser ce programme, et ne fait pas la distinction entre les grandes banques de terminologie et les petites bases parfois hautement structurées et visant à un accès à des connaissances factuelles plutôt que linguistiques (cf. Blampain 
1992). L'aspect qu'il conteste est l'accessibilité de ces informations. Il est effectivement peu raisonnable de supposer que différentes catégories d'utilisateurs seront en mesure d'extraire ces connaissances, sans au moins supposer une typologie des usagers, donc des fonctions. Il les schématise ainsi, s’inspirant de Wiegand:

1. reception of L1-texts

2. production of L1-texts

3. reception of L2-texts

4. production of L2-texts

5. L1-L2 translation

6. L2-L1 translation

7. acquisition of encyclopedic information

8. acquisition of linguistic information

Bergenholtz et Kaufmann 1997: 99.

Bergenholtz, en tant que co-auteur d'un dictionnaire de génie génétique (Kaufmann et Bergenholtz 1992), a tenté de mettre en pratique cette démarche. On remarque plusieurs innovations par rapport aux dictionnaires classiques, d'orientation terminologique ou non.

- Traitement différencié du danois et de l'anglais.

En effet, le lecteur est Danois. Il a une certaine connaissance de l'anglais mais il préfère se renseigner dans sa langue maternelle. Les informations encyclopédiques sont donc indiquées en danois, les informations linguistiques surtout en anglais, car l'utilisateur aura sans doute à lire des textes anglais, mais peut-être aussi à rédiger en anglais.

- Définitions «de vulgarisation» plutôt que pour le spécialiste.

- Exemples non seulement pertinents mais aussi, selon l'auteur, «intéressants, parlants, typiques, vrais».

On peut se demander si le procès intenté par Bergenholtz aux terminologues a des chances d'aboutir. Puisque c'est le point de vue des utilisateurs qui nous intéresse ici, nous nous bornerons à évoquer cet aspect des choses. Il est possible d'objecter, contrairement à ce qu'affirme Bergenholtz, que la terminologie est davantage tournée vers ces finalités que la lexicographie. En effet, si l'on ne fait guère la distinction entre la terminologie et la terminographie, c'est en partie parce que la terminologie prend toujours la forme d'un produit terminographique.

Malgré le ton polémique souvent employé, il est possible de trouver des terminologues qui sont tout à fait d'accord avec le diagnostic de Bergenholtz, même s'ils ne partagent pas son avis sur les solutions. Les principaux opposants à une terminologie divorcée des besoins des utilisateurs se sont réunis au sein du groupe Terminologie et intelligence artificielle, qui a déjà organisé trois colloques (Villetaneuse en 1995, Toulouse en 1997 et Nantes en 1999). Or, lors d'une de ces rencontres, deux des principaux organisateurs, Didier Bourigault et Monique Slodzian, ont publié un manifeste sous forme de tutorial, désormais disponible dans Terminologies nouvelles 18.

Ils commencent par expliquer que la terminologie connaît de très nombreuses applications, qui correspondent chacune à un besoin particulier :

- thésaurus (indexation automatique)

- index structurés (documentation électronique) 
- terminologies de référence (aide à la rédaction)

- référentiels terminologiques (gestion des données techniques)

- ontologies (mémoires d'entreprises, aides à la décision)

- réseaux lexicaux spécialisés (recherche thématique toile sur la toile)

- glossaires de référence (outils de communication internes ou externes)

- bases de connaissances terminologiques (corpus de référence)

Bourigault et Slodzian 1999: 29.

Il est évident que parmi ces produits beaucoup ne correspondent pas à ce que nous pensons être le dictionnaire prototypique, mais c'est précisément cette variété qui caractérise le dictionnaire de l'avenir.

En plus de la multiplicité des produits, les collègues contestent la terminologie idéale, unique, qui préexiste dans les faits, et qui ne demande qu'à être transcrite sous forme de dictionnaire.

"C'est ainsi que s'impose le constat de la variabilité des terminologies; étant donné un domaine d'activité, il n'y a pas UNE terminologie, qui représenterait LE savoir sur le domaine, mais autant de terminologies que d'applications dans lesquelles ces terminologies ont été utilisées. Ces terminologies different quant aux unités retenues et à leur description selon l'application visée. Par ailleurs, la croissance terminologique, induite par la prolifération de tous sens de connaissances, entraîne la nécessité de mises à jour permanentes si l'on veut répondre aux besoins des utilisateurs. » Bourigault et Slodzian 1999: 30.

$\mathrm{Au}$ contraire:

"La tâche d'analyse terminologique n'est donc pas un exercice de redécouverte d'un système notionnel préexistant qui caractériserait le domaine» Bourigault et Slodzian 1999: 31.

Que doit donc faire le terminologue?

"L'expérience montre en effet qu'une terminologie élaborée pour une application à un moment donné n'est jamais identique à celle construite pour une application différente. Ces limites fortes à la réutilisabilité n'excluent pas des relations d'inclusion ou de chevauchements partiels entre terminologies dédiées à des applications différentes dans un même domaine d'activité. » Bourigault et Slodzian 1999: 30.

Ce constat est dur pour ceux qui ont plaidé pour la portabilité de la terminologie, car il laisse supposer qu'il convient de repartir à zéro chaque fois qu'on élabore une nouvelle terminologie, mais il existe des moyens de recyclage, de réutilisation des terminologies déjà constituées que nous n'aurons guère le temps d'évoquer ici (mais voir à ce sujet Chrobot 1999).

"Pour chaque unité choisie, l'analyste construit une signification (type) à partir des sens (occurrences) attestés dans le corpus. Dans cette tâche, il est guidé en amont par le corpus (spécificités lexicales) et en aval par l'application (utilisation des descriptions).» Bourigault et Slodzian 1999: 30.

Les terminologues sont-ils prêts à relever le défi ? Ils nous semble que c'est déjà le cas, et nous aimerions citer trois exemples de produits terminologiques (pour ne pas dire dictionnaires) qui sont exemplaires du point de vue de la prise en compte de l'utilisateur.

Le premier est un exemple somme toute assez banal de terminologie élaborée pour une organisation internationale et donc multilingue, mais il est typique du dialogue qui s'instaure entre concepteur et utilisateur. Il s'agissait de préparer un lexique 
bilingue, français-anglais, pour la Cour européenne des droits de l'Homme. Christine Chodkiewicz venait de terminer la traduction d'un livre sur les droits de l'Homme, et ne trouvant pas de terminologie déjà constituée, elle s'était mise en rapport avec la Cour pour savoir si elle en disposait d'une. La réponse a été négative, mais le service de traduction lui a fait savoir que, si elle en faisait une, les traducteurs seraient preneurs. Après avoir contacté le CTN, elle a décidé de travailler à partir d'un corpus tiré de la production écrite de la Cour (la Déclaration européenne des droits de l'Homme et un certain nombre d'arrêts). On sait que ces arrêts sont rédigés indifféremment en français ou en anglais, puis traduits dans l'autre langue. De même, il existe des versions de langues anglaise et française de la Déclaration des droits de l'Homme. D’un point de vue juridique, les deux versions sont considérées comme équivalentes et officielles.

Non seulement la terminologie visée devait correspondre à celle que la Cour souhaitait, mais de plus elle devait être confectionnée à partir des productions textuelles de la Cour même, dépouillées automatiquement dans un premier temps grâce au logiciel LEXTER (voir Bourigault, Chodkiewicz et Humbley 1999). D'ailleurs les juristes se sont déclarés très satisfaits de la qualité des textes dans les deux langues et incapables de distinguer l'original de la traduction.

La production écrite de la Cour représente donc le corpus idéal pour l'extraction terminologique. Misant sur la récurrence des termes, l'équipe a pu proposer à la Cour un lexique bilingue très classique, mais qui représente non pas la terminologie des droits de l'Homme, mais les termes employés par l'utilisateur, et qu'il est susceptible de réemployer. Une deuxième version sera réalisée à la lumière de la réaction des traducteurs, et une présentation de type hypertexuel, exploitant l'interface d'utilisation de LEXTER et permettant un accès direct aux textes où les termes sont employés, est envisagée à plus long terme. Le dictionnaire est donc le fruit d'un long dialogue avec le destinataire, ce qui n'empêche pas de voir d'autres finalités, notamment son exploitation par d'autres tribunaux et la prise en compte de sa nomenclature pour la traduction de textes juridiques dans les langues des pays candidats à l'Union européenne.

Le deuxième exemple concerne une autre application juridique, cette fois-ci dans le domaine des droits... d'auteur. Nous savons que les accords internationaux sur la libéralisation du secteur tertiaire butent depuis très longtemps sur les épineux problèmes de droits d'auteur. Ces problèmes sont particulièrement compliqués. D'une part, il s'agit de protéger les droits légitimes des auteurs de différents ordres, et au delà de protéger le patrimoine culturel, mais d'autre part, il s'agit aussi, très concrètement, de comprendre les législations nationales et internationales très complexes et souvent incompatibles. Voilà pourquoi droit d'auteur n'égale pas copyright - il s'agit de deux concepts juridiques différents. Il faut donc d'abord voir clair pour pouvoir prendre des mesures efficaces. C'est dans cet esprit qu'une équipe de juristes «Droit des systèmes d'information et de communication» (DISC) du Centre d'études sur la coopération juridique internationale, sous l'impulsion d'Isabelle de Lamberterie, a décidé de faire une liste des termes du domaine des droits d'auteur/ copyright, et d'élaborer des définitions, soit un travail de lexicographie à l'ancienne, comme les scribes qui notaient leur propre glossaire. La responsable avait réuni des juristes spécialistes du droit français, belge, anglais, américain, canadien et international, qui ont d'abord fait une liste des termes. Ensuite ils ont commencé à remplir 
des fiches qu'elle avait conçues selon un modèle terminologique classique. C'est au moment de les collationner et de les comparer que l'équipe de coordination s'est rendu compte qu'il y avait un problème: les fiches étaient si complètes, si compliquées, qu'il était difficile pour les juristes non pas de les comprendre mais de les présenter de façon claire aux non-juristes qui doivent s'occuper de droit d'auteur sur le plan international. Chaque fiche comporte la présentation d'un concept du domaine tel qu'il existe dans le droit du pays concerné, et se divise en cinq parties: une définition-analyse, la source légale de la définition, les autres sources (doctrine, jurisprudence, pratique, etc.), les principaux textes de référence, et des exemples, et peut comporter plusieurs pages. La définition-analyse, à elle seule, comporte plusieurs paragraphes et porte sur les faits saillants du concept dans le système juridique concerné, qui peuvent être très différents d'un pays à l'autre.

C'est alors qu'une terminologue, Véronique Alory, est intervenue. Sa première démarche a été d'informatiser les fiches déjà existantes (c'est la fonction terminoticienne déjà mentionnée), ce qui a nécessité une restructuration, et puis elle a commencé le travail de comparaison des concepts. Cette mise à plat s'est faite à l'aide de grilles très simples, mais très systématiques, qui donnent un aperçu synoptique, et qui permettent d'établir des comparaisons lorsqu'il est possible et de constater des lacunes. Pour le tableau de synthèse comparative du concept artiste interprète, par exemple (voir fig. 1, où seuls les droits français, belge et britannique sont présentés), on constate que pour le droit français, un artiste interprète est un comédien, un chanteur, un musicien, un danseur, un artiste de variétés ou un artiste de cirque. On a en quelque sorte une définition par extension. Le droit belge, dans ce cas précis, est une copie conforme de son homologue français, mais le britannique est bien différent: l'artiste interprète n'est ni comédien, ni danseur, ni artiste de cirque; la définition-analyse actuelle ne permet pas de savoir s'il peut être un chanteur ou un artiste de variétés. D'autres critères ou caractères sont également traduits sous forme de tableau de synthèse comparative, permettant ainsi une comparaison plus complète.

FIGURE 1

Synthèse comparative du concept artiste interprète

\begin{tabular}{|l|c|c|c|c|}
\hline & droit français & droit belge & droit britannique & etc. \\
\hline Comédien & OUI & OUI & NON & \\
\hline Chanteur & OUI & OUI & & \\
\hline Musicien & OUI & OUI & OUI & \\
\hline Danseur & OUI & OUI & NON & \\
\hline Artiste de variétés & OUI & OUI & & \\
\hline Artiste de cirque & OUI & OUI & NON & \\
\hline
\end{tabular}

Face à cette présentation de leur réalité, la réaction des juristes était mitigée; ils étaient contents d'avoir un outil de travail, mais réservés quant à l'idée de se servir de grilles pour la présentation à des non-juristes. On peut tirer plusieurs conclusions de ce travail, qui n'est pas encore terminé. La première, c'est que les approches terminologiques peuvent se compléter - une orientation plutôt lexicographique a 
été corrigée par l'intervention d'une terminologue plutôt cognitiviste. Et contrairement à ce que prétend Bergenholtz, c'est la terminologue qui permet au public plus large d'accéder à la connaissance spécialisée La seconde, c'est que deux formes d'intervention terminologique ont été nécessaires afin de trouver la solution qui convenait le mieux aux utilisateurs; toutes ne ressemblent pas au dictionnaire classique.

Le troisième exemple est plutôt une constellation de cas d'utilisation d'une démarche terminologique dans un contexte d'apprentissage. Le premier peut être caractérisé par le schéma que nous adaptons d'une présentation donnée par H. Picht (1997: 37). Il s'agit d'appliquer les principes de la terminologie la plus classique à une présentation de concepts spécialisés visant à l'initiation des étudiants aux techniques de la banque, l'aspect retenu ici étant le chèque.

\section{FIGURE 2}

\section{Connaissances terminologiques liées au concept de chèque}

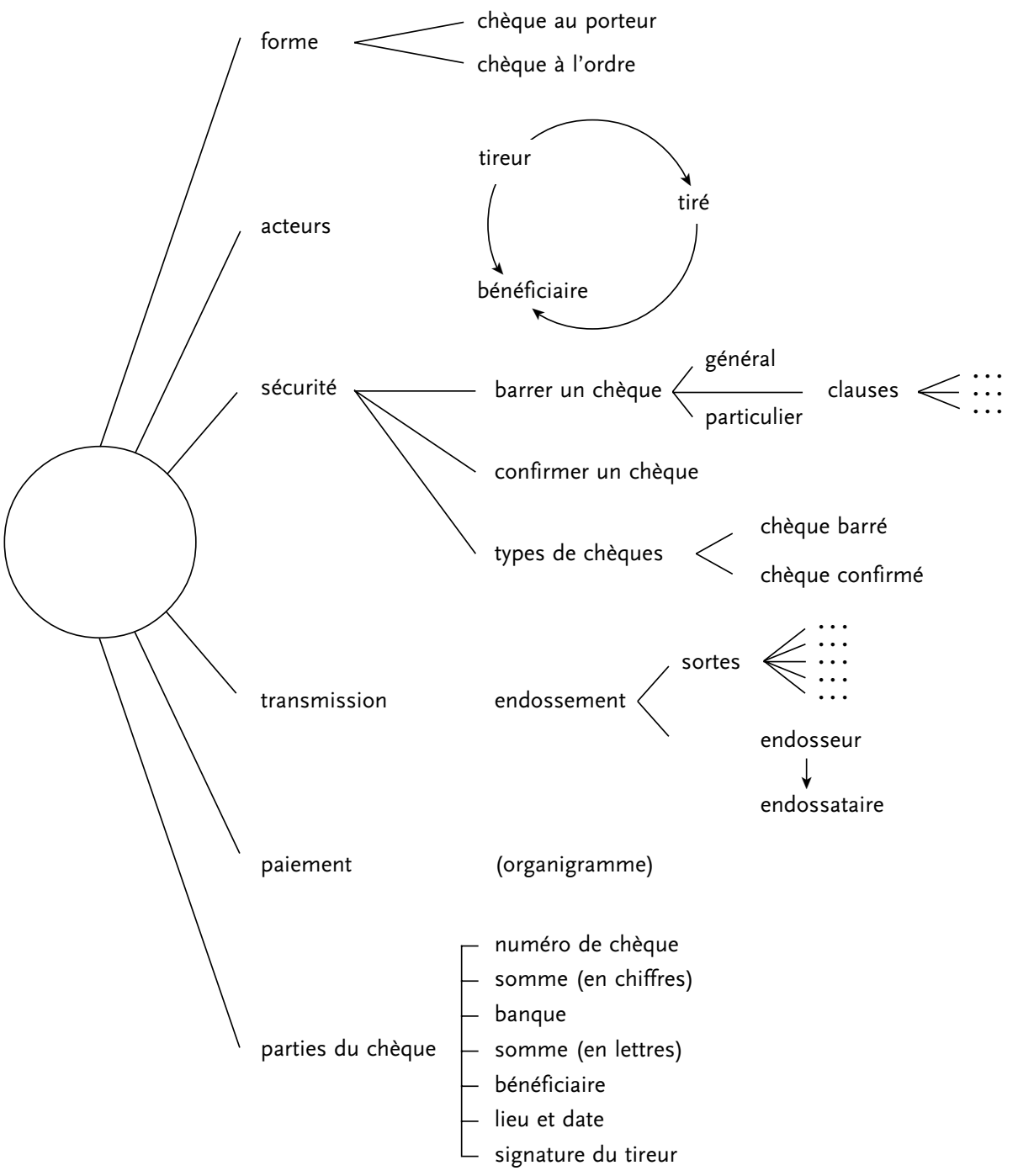


On remarque que le schéma reproduit aussi bien les relations généralement privilégiées en terminologie (relations logiques: les formes, les types de chèques; relations partitives: les parties du chèque), ainsi que d'autres moins fréquemment invoquées (relations de transmission), sans oublier les acteurs et les actions associés. Ce schéma n'est certes pas un dictionnaire, mais le fruit du travail du terminologue dont le rôle est de faciliter l'accès par les mots aux connaissances spécialisées. Il sert donc directement à ces deux types d'utilisateurs, aux étudiants et à leurs professeurs.

Un autre exemple pédagogique, déjà publié (Humbley 1996), concerne une situation similaire, où des étudiants de langues étrangères appliquées (LEA) devaient s'initier aux méthodes des assurances. Le même genre de grille de caractères que Véronique Alory a utilisée pour les droits d'auteur a été adapté aux différents types d'assurance. Cette grille ne rend pas compte de tous les aspects des assurances, mais elle permet aux étudiants de visualiser les différences entre entités comparables et de mieux comprendre les textes qu'ils ont déjà étudiés avec les professionnels. Mieux, ces grilles ne sont pas l'apanage du professeur, car une fois maîtrisées, elles servent dans d'autres contextes; les étudiants ont su les adapter lorsqu'ils ont découvert l'existence d'autres catégories d'assurance-vie temporaire, qui n'étaient pas mentionnées dans leurs premiers documents. Comme dans l'exemple précédent, le produit terminologique n'est pas un dictionnaire, ni même un glossaire, mais surtout une méthode.

On relève plusieurs exemples de ce genre d'exploitation pédagogique dans Terminologies nouvelles 17.

Il est à souligner que dans plusieurs des cas que nous citons l'utilisateur intervient non seulement après coup, comme dans les exemples donnés par P. Bogaart, mais aussi avant et pendant le travail lexicographique ou terminologique. L'utilisateur participe donc à la conception et à la réalisation du dictionnaire ou de la terminologie. On peut se demander si on ne boucle pas la boucle des moines copistes, usager et producteur en même temps. Mais c'est plutôt une spirale et une spirale ascendante. La division du travail se poursuit, s'accentue, mais impose davantage de collaboration. Le terminologue ou le lexicographe intervient donc mais de façon modulaire. Pour H. K. Simonsen (à paraître), les utilisateurs d'une banque de terminologie d'une grande entreprise de télécommunications (Tele Danmark A/S) sont des «active fellow players». Le concepteur de la banque, qu'il caractérise comme un «lexicographic knowledge management system», prône le «user involvement», qui aboutit à une démocratie lexicographique, peut-être plus réaliste que la «bottom up lexicography» évoquée par Carr en 1997. Plus prosaïquement on pourrait parler de dictionnaires et de terminologie participatifs. Et ce n'est pas complètement nouveau. Après tout, les grandes banques de terminologie - BTQ (devenue depuis le Grand dictionnaire terminologique), Termium, Eurodicautom - sont des terminologies faites par et pour les utilisateurs. Les exemples donnés ici intègrent la participation des utilisateurs à des degrés et à des stades variables.

En conclusion, on ne peut pas faire l'économie d'une réflexion sur ce que sont, en l'an 2000, les objets dictionnaires et terminologies. On constate que le lexicographe et le terminologue disposent de nouvelles possibilités, notamment pour la constitution et l'exploitation de corpus et pour la mise en rapport des données recueillies. Par ailleurs, on reconnaît de nouveaux besoins liés à l'essor de l'Internet, aux exigences du multilinguisme, du moins en Europe, à l'accroissement phénoménal de la quantité 
d'informations à gérer et à la nécessité d'adapter la pédagogie à des formes d'enseignement plus autonomes. Cette nouvelle donne nécessite un réexamen en profondeur de ce que terminologues et lexicographes peuvent apporter à la résolution de ces problèmes de communication. Ils y arriveront s'ils écoutent bien l'utilisateur.

\section{RÉFÉRENCES}

Bergenholtz, H. (1995) : «Wodurch unterscheidet sich Fachlexikographie von Terminographie?» Lexicographica, II p. 50- 59.

Bergenholtz, H. et S. TARP (1993): Manual i fagleksikografi: Udarbejdelse af fagordboeger: problemer og losningforslag [Manuel de lexicographie de spécialité; élaboration de dictionnaires techniques — problèmes et solutions suggérées], Forlaget Systime a/s, Herning.

Bergenholtz, H. et U. Kaufmann (1997): «Terminography and lexicography. A critical survey of dictionaries from a single specialised field», Hermes, 18, p. 91-125.

Blampain, D. (1992): «Traduction et écosystèmes terminologiques», Terminologie et traduction, 2/3, p. 457-466.

BogaARds, P. (1993): «Dictionary use: a state-of-the art statement», aila '93, 13/08/1993, section papers, p. 697.

Bourigault, D. et M. Slodzian (1999): «Pour une terminologie textuelle», Terminologies nouvelles 19 , p. 29-32.

Bourigault, D., Chodkiewicz, C. et J. Humbley (1999): «Construction d'un lexique bilingue des droits de l'homme à partir de l'analyse automatique d'un corpus aligné », Terminologies nouvelles 19, p. $70-77$.

CARR, M. (1997) : «Internet dictionaries and lexicology», International Journal of Lexicography, 10, p. 209-230.

Снговот, А. (1999): «Enrichissement terminologique en anglais fondé sur des dictionnaires généraux et spécialisés», Terminologies nouvelles 19, p. 78-88.

Humbley, J. (1996): «Terminological methods as an aid to learning a new subject in a multilingual setting: examples in the field of insurance", in BUDIN, Multilingualism in specialist communication, Proceedings of the 10th European LSP Symposium, Vienna, September 1995, Vienne, IITF. p. 1011-1020.

JАковSEN, A.L. (1992): Oversaettelse af fagsproglige tekster, ARK 65. Sproginstituternes Arbejdspapir, Handelshojskolen i Kobenhavn.

Kaufmann, U. et H. Bergenholtz (1992): Genteknologisk ordbog, Copenhague. Gad.

Ріснт, Н. (1997) : «Erarbeitung und Anwendung begrifflicher Strukturierungen », Hermes (Handelshojskolen i Aarhus) 18. p. 33-50.

Rint (1993), Terminologies nouvelles 10: la phraséologie.

- (1998), Terminologies nouvelles 17: l'enseignement de la terminologie.

Simonsen, H.K. (à paraître), «Report on the Design and Development of a Lexicographic Convergence Telecom Lexinome» dans EURALEX 2000. 